\title{
Electroacoustic Waves Excited by a Space Vehicle in Ionized Atmosphere and Its Effect on Radar Return
}

\author{
Kun-Mu Chen
}

\begin{abstract}
Contribution From the Department of Electrical Engineering, Michigan State University, East Lansing, Mich.
\end{abstract}
(Received August 31, 1964)

\begin{abstract}
When a conducting space vehicle in the ionosphere is illuminated by an incident electromagnetic wave from the ground, a current and charge are induced on the vehicle. The induced current and charge in turn generate two scattered fields in the ionosphere; the electromagnetic (EM) and an electroacoustic (EA) wave. A part of the EA wave is converted to an EM wave across the discontinuity of the ionosphere and reaches the ground. As the result, the radar return of a space vehicle in the ionosphere is greatly enhanced. In this paper, the case of a conducting cylinder in a plasma and illuminated by an EM wave is considered. The induced current and charge on the cylinder are determined and the scattered EM and EA waves are calculated. The corresponding radar cross sections due to EM and EA waves are defined. It is shown that the enhancement of the radar return due to an EA wave bears some resemblances to the large outbursts of the reflected HF signals from the satellites observed by Kraus.
\end{abstract}

\section{Introduction}

When an oscillating electric source is placed in a compressive plasma an electromagnetic (EM) and an electroacoustic (EA) wave are generated. Recently, Cohen [1962] has shown the possible excitation of an EA wave by a radiating source. Hessel and Shmoys [1962] have studied the case of a hertzian dipole in a compressive plasma. Chen [1963] has investigated the effect of an EA wave on the radiation of a dipole antenna in a lossless and a lossy compressive plasma. Wait [1964 a, b] has also studied the problem of an antenna in a compressive plasma. In these studies they all find the significant effect of an EA wave on the radiation of a radiating source.

When a conducting space vehicle moving in the ionized atmosphere is illuminated by an incident EM wave from the ground, a current and charge are induced on the vehicle. The oscillating current and charge on the vehicle can excite a scattered EA wave in addition to a scattered EM wave. A part of the EA wave converts to an EM wave across the density discontinuities and reaches the ground. As the result, the radar return of a conducting space vehicle in a compressive plasma is greatly enhanced. In this paper a space vebicle is assumed to have the shape of a thin cylinder and we calculate the effect of the excited EA wave on its radar return.

Kraus [1958; Kraus, Higgy, and Albus, 1958; Kraus, Higgy, and Crone, 1960] have reported some unusual observations of the $\mathrm{CW}$ reflected HF signal from the satellites at Ohio State Observatory. They observed that the radar returns from the satellites consists of a group of very strong bursts. The appearance of the group of bursts is like a series of regularly spaced spikes with the largest bursts occurring near the center of the group and decreasing in amplitude from the middle to the both sides of the group.

The enhancement of the radar return from a conducting cylinder in a compressive plasma due to an EA wave is shown to have a similar nature as that observed by Kraus. This may help the explanation of those unusual observations.

\section{Statement of Problem and Basic Equations}

The geometry of the problem is shown in figure 1. A cylinder in the ionosphere which is a compressive plasma medium is assumed to be perfectly conducting. A plane EM wave from the ground is incident on the cylinder. The problem is (1) to find the induced current and charge on the cylinder, (2) to calculate the scattered EM and EA waves generated by the induced current and charge on the cylinder, (3) to find the radar cross sections of the cylinder due to the EM and EA waves and (4) to study the conversion of an EA wave to an EM wave across the density discontinuity of the ionosphere. 


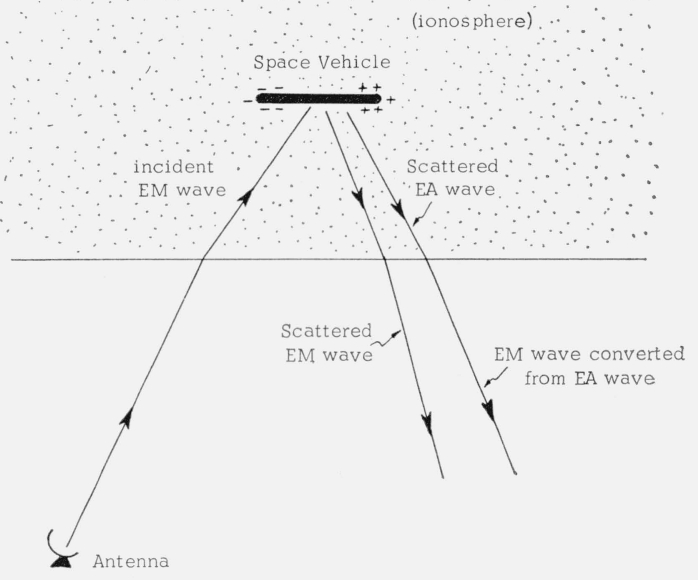

FIgURE 1. Waves incident on and scattered by a space vehicle in the ionosphere.

In our problem, the plasma is assumed to be homogeneous and neutral and the perturbation of the plasma due to electric charges on the cylinder is also assumed to be sufficiently small for linearized equations to be valid. The plasma is assumed to be lossless and no static electric or static magnetic field is assumed to be present. Under these assumptions the basic equations are Maxwell equations and linearized Euler equations as follows:

$$
\begin{gathered}
\nabla \times \vec{E}=-\mu_{0} \frac{\partial H}{\partial t} \\
\nabla \times \vec{H}=\overrightarrow{J^{s}}-e n_{0} \vec{V}+\epsilon_{0} \frac{\partial \vec{E}}{\partial t} \\
\nabla \cdot \vec{E}=\frac{\rho^{s}}{\epsilon_{0}}-\frac{e n_{1}}{\epsilon_{0}} \\
\nabla \cdot \vec{H}=0 \\
n_{0}(\nabla \cdot \vec{V})+\frac{\partial n_{1}}{\partial t}=0 \\
n_{0} m\left(\frac{\partial \vec{V}}{\partial t}\right)=-n_{0} e \vec{E}-m v_{0}^{2} \nabla n_{1} .
\end{gathered}
$$

In the above notation, $\vec{E}$ and $\vec{H}$ are the electric and magnetic fields. $\overrightarrow{J^{s}}$ and $\rho^{s}$ are the electric current and charge densities. $n_{0}$ and $n_{1}$ are the ambient electron density and the perturbation of the electron density. $\vec{V}$ and $v_{0}$ are the mean induced velocity and the rms velocity of electrons. $e$ and $m$ are the charge and mass of the electron. The motion of positive ions in the plasma is ignored. The time dependence factor for all fields is assumed to be $e^{j \omega t}$.
Following Chen's analysis, the fields can be separated into the EM and EA modes as follows:

For the EM mode $\left(\vec{E}_{e}, \vec{H}, \vec{V}_{e}\right)$

$$
\begin{aligned}
\nabla \times \vec{E}_{e} & =-j \omega \mu_{0} \vec{H} \\
\nabla \times \vec{H} & =\vec{J}^{s}-e n_{0} \vec{V}_{e}+j \omega \epsilon_{0} \vec{E}_{e} \\
\nabla \cdot \vec{E}_{e} & =\frac{\rho^{s}}{\epsilon_{0} \xi} \\
\nabla \cdot \vec{H} & =0
\end{aligned}
$$

and

$$
\xi=1-\frac{\omega_{p}^{2}}{\omega^{2}}
$$

with

$$
\omega_{p}^{2}=\frac{n_{0} e^{2}}{\epsilon_{0} m}
$$

For the EA mode $\left(\vec{E}_{a}, \vec{V}_{a}, n_{1}\right)$,

$$
\begin{gathered}
\nabla \times \vec{E}_{a}=0 \\
j \omega \epsilon_{0} \vec{E}_{a}-e \vec{n}_{0} \vec{V}_{a}=0 \\
j \omega \vec{V}_{a}=-\frac{e}{m} \vec{E}_{a}-\frac{v_{0}^{2}}{n_{0}} \nabla n_{1} .
\end{gathered}
$$

In this separation of modes, the relations of $\vec{E}=\vec{E}_{e}+\vec{E}_{a}$ and $\vec{V}=\vec{V}_{e}+\vec{V}_{a}$ are valid. The EM mode consists of an electric and a magnetic field but no accumulation of charges. The EA mode consists of an electric field and an accumulation of charges but no magnetic field. The details of the analysis are omitted here for brevity.

The $\vec{E}_{e}$ field of the EM mode can be obtained from

$$
\overrightarrow{E_{e}}=-\nabla \phi \rightarrow j \omega A
$$

where

$$
\begin{aligned}
& \vec{A}=\frac{\mu_{0}}{4 \pi} \int \vec{J}^{s} \frac{e^{-j \beta_{e} r}}{r} d v \\
& \phi=j \frac{1}{\omega \epsilon_{0} \mu_{0} \xi} \nabla \cdot \vec{A} \\
& \beta_{e}=\omega \sqrt{\epsilon_{0} \mu_{0} \xi}=\frac{\sqrt{\omega^{2}-\omega_{p}^{2}}}{c},
\end{aligned}
$$

$\vec{A}$ and $\phi$ are the vector and scalar potentials, and $c$ is the velocity of light.

The $\vec{E}_{a}$ field of the EA mode can be obtained from

$$
\vec{E}_{a}=\frac{e v_{0}^{2}}{\left(\omega^{2}-\omega_{p}^{2}\right) \epsilon_{0}} \nabla n_{1}
$$


where

$$
\begin{aligned}
& n_{1}=\frac{1}{4 \pi} \frac{\omega_{p}^{2}}{e v_{0}^{2}} \int \rho^{s} \frac{e^{-j \beta_{a} \tau}}{r} d v \\
& \beta_{a}=\frac{\sqrt{\omega^{2}-\omega_{p}^{2}}}{v_{0}} .
\end{aligned}
$$

From (17) and (21), it is evident that once the electric current and charge induced on the cylinder are determined all fields can be calculated.

\section{Induced Current and Charge on Cylinder}

As shown in figure 2, a conducting cylinder with a length of $2 h$ and a radius of $a$ is illuminated by a plane EM wave at an angle $\theta$. The length of the cylinder is assumed to be small compared with the wavelength of the incident EM wave and $a$ is assumed to be much smaller than $h$.

The tangential component of the incident $\vec{E}$ field along the cylinder is assumed to be

$$
\left[E_{i}\right]_{z}=E_{0} \cos \theta e^{-j \beta_{e} \sin \theta z}
$$

where $E_{0}$ is a constant.

The current and charge on the cylinder maintains a tangential electric field at the surface which can be expressed as

$$
E_{z}=\left[E_{e}\right]_{z}+\left[E_{a}\right]_{z}
$$

where $\left[E_{e}\right]_{z}$ is the electric field of the EM mode and $\left[E_{a}\right]_{z}$ is that of the EA mode. From (16) and (18),

$$
\left[E_{e}\right]_{2}=-j \frac{\omega}{\beta_{e}^{2}}\left(\frac{\partial^{2}}{\partial z^{2}}+\beta_{e}^{2}\right) A_{z}
$$

where $A_{2}$ is the tangential component of the vector potential at the surface of cylinder. From (20),

$$
\left[E_{a}\right]_{z}=\frac{e v_{0}^{2}}{\left(\omega^{2}-\omega_{p}^{2}\right) \epsilon_{0}} \frac{\partial}{\partial z} n_{1}
$$

Since the tangential electric field should vanish at the surface of cylinder we obtain the following equation.

$$
\left[E_{e}\right]_{z}+\left[E_{a}\right]_{z}=-\left[E_{i}\right]_{z}
$$

If the cylinder is very thin compared with the wavelength of the incident EM wave, only axial current $I_{z}$ is induced on the cylinder. The charge on the cylinder is related to $\bar{I}_{z}$ by the equation of continuity as

$$
q=j \frac{1}{\omega} \frac{\partial}{\partial z} I_{z}
$$

The substitution of (17), (21), (28), (23), (25),

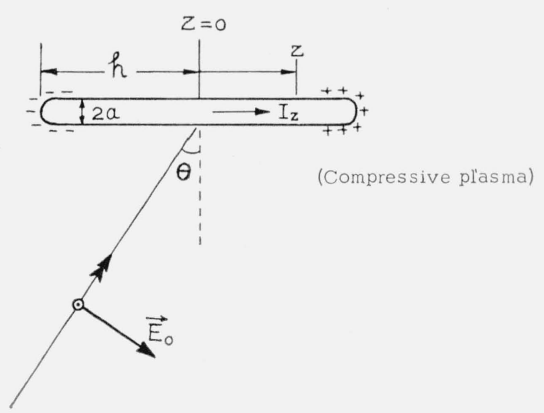

Figure 2. Cylinder illuminated by a plane EM wave at an angle $\theta$.

and (26) in (27) gives an integral equation for $I_{z}$ as

$$
\begin{aligned}
& \left(\frac{\partial^{2}}{\partial z^{2}}+\beta_{e}^{2}\right) \int_{-h}^{h} I_{z}\left(z^{\prime}\right) K_{e}\left(z, z^{\prime}\right) d z^{\prime} \\
& -\frac{\omega_{p}^{2}}{\omega^{2}} \frac{\partial}{\partial z} \int_{-h}^{h} \frac{\partial I\left(z^{\prime}\right)}{\partial z^{\prime}} K_{a}\left(z, z^{\prime}\right) d z^{\prime} \\
& =-\frac{j 4 \pi \beta_{e}^{2}}{\omega \mu_{0}} E_{0} \cos \theta e^{-j \beta_{e} \sin \theta z}
\end{aligned}
$$

where the kernels $K_{e}\left(z, z^{\prime}\right)$ and $K_{a}\left(z, z^{\prime}\right)$ can be expressed as

$$
\begin{aligned}
& K_{e}\left(z, z^{\prime}\right)=\frac{\exp \left[-j \beta_{e} \sqrt{\left(z-z^{\prime}\right)^{2}+a^{2}}\right]}{\sqrt{\left(z-z^{\prime}\right)^{2}+a^{2}}} \\
& K_{a}\left(z, z^{\prime}\right)=\frac{\exp \left[-j \beta_{a} \sqrt{\left.\left(z-z^{\prime}\right)^{2}+a^{2}\right]}\right.}{\sqrt{\left(z-z^{\prime}\right)^{2}+a^{2}}} .
\end{aligned}
$$

The induced current $I_{z}$ can be determined by solving (29). Since an exact solution for $I_{z}$ is too difficult to obtain we determine $I_{z}$ approximately with an iterative method. From (19) and (22) it is evident that

$$
\frac{\beta_{a}}{\beta_{e}}=\frac{c}{v_{0}}>>1 .
$$

This means that $K_{a}\left(z, z^{\prime}\right)$ is a much more rapidly oscillating kernel than $K_{e}\left(z, z^{\prime}\right)$. As the result the second term of the left-hand side of (29) is much smaller than the first term. Therefore, for the zeroth order solution of $I_{z}$ the following equation is to be solved.

$$
\begin{aligned}
\left(\frac{\partial^{2}}{\partial z^{2}}+\beta_{e}^{2}\right) \int_{-h}^{h}\left[I_{z}\left(z^{\prime}\right)\right]_{0} & K_{e}\left(z, z^{\prime}\right) d z^{\prime} \\
& =-\frac{j 4 \pi \beta_{e}^{2}}{\omega \mu_{0}} E_{0} \cos \theta e^{-j \beta_{e} \sin \theta z}
\end{aligned}
$$

where $\left[I_{z}\left(z^{\prime}\right)\right]_{0}$ stands for the zerotb order solution of $I_{z}$. Equation (33) is solved in appendix for the case of $h<<\lambda_{e}$ where $\lambda_{e}$ is the wavelength of incident 
EM wave. The solution of $\left[I_{z}\left(z^{\prime}\right)\right]_{0}$ can be summarized as

$$
\begin{aligned}
{\left[I_{z}(z)\right]_{0} } & =C E_{0} \cos \theta\left(\cos \beta_{e} z-\cos \beta_{e} h\right) \\
C & =\frac{j 4 \pi}{\omega \mu_{0}}\left[\frac{1-\cos \beta_{e} h}{\cos \beta_{e} h T_{1}-T_{2}}\right] \\
T_{1} & =\int_{-h}^{h}\left(\cos \beta_{e} z^{\prime}-\cos \beta_{e} h\right) K_{e}\left(0, z^{\prime}\right) d z^{\prime} \\
T_{2} & =\int_{-h}^{h}\left(\cos \beta_{e} z^{\prime}-\cos \beta_{e} h\right) K_{e}\left(h, z^{\prime}\right) d z^{\prime} .
\end{aligned}
$$

Thus the induced current on the cylinder is determined as a function of cylinder size $(h, a)$, the amplitude $\left(E_{0}\right)$ and the incident angle $(\theta)$ of the incident EM wave.

The charge on the cylinder can be determined from (28) and (34) as

$$
[q(z)]_{0}=-j \frac{\beta_{e}}{\omega} C E_{0} \cos \theta \sin \beta_{e} z .
$$

The zeroth order solutions for $I_{z}$ and $q$ are quite sufficient and convenient for further development of the theory. In case the first order solution is needed the following equation is to be solved.

$$
\begin{aligned}
\left(\frac{\partial^{2}}{\partial z^{2}}+\beta_{e}^{2}\right) & \int_{-h}^{h}\left[I_{z}\left(z^{\prime}\right)\right]_{1} K_{e}\left(z, z^{\prime}\right) d z^{\prime} \\
& =-\frac{j 4 \pi \beta_{e}^{2}}{\omega \mu_{0}} E_{0} \cos \theta e^{-j \beta_{e} \sin \theta z} \\
& +\frac{\omega_{p}^{2}}{\omega^{2}} \frac{\partial}{\partial z} \int_{-h}^{h} \frac{\partial}{\partial z^{\prime}}\left[I_{z}\left(z^{\prime}\right)\right]_{0} K_{a}\left(z, z^{\prime}\right) d z^{\prime},
\end{aligned}
$$

$\left[I_{z}\left(z^{\prime}\right)\right]_{1}$ is the first order solution of $I_{z}$ and $\left[I_{z}\left(z^{\prime}\right)\right]_{0}$ is expressed in (34).

For simplicity, (34) and (38) are used in the calculation of the scattered EM and EA waves in the next section.

\section{Scattered EM and EA Waves}

For the practical interest, we calculate the backscattered EM and EA waves at a distant point from the cylinder and in the direction of $\theta$.

To calculate the backscattered EM wave at a point $\left(R_{0}, \theta\right)$ from the center of the cylinder, the vector potential $\vec{A}$ in (17) is evaluated first. With the current on the cylinder expressed as in (34) we have

$$
\begin{aligned}
A_{z}= & \frac{\mu_{0}}{4 \pi} \frac{e^{-j \beta_{e} R_{0}}}{R_{0}} C E_{0} \cos \theta \int_{-h}^{h}\left(\cos \beta_{e} z-\cos \beta_{e} h\right) e^{j \beta_{e} z \sin \theta} d z \\
= & \frac{\mu_{0} e^{-j \beta_{e} R_{0}}}{2 \pi \beta_{e} R_{0} \cos \theta} C E_{0}\left[\sin \beta_{e} h \cos \left(\beta_{e} h \sin \theta\right)\right. \\
& \left.-(\sin \theta+\cos \theta \cot \theta) \cos \beta_{e} h \sin \left(\beta_{e} h \sin \theta\right)\right] .
\end{aligned}
$$

The electric field of the scattered EM wave which is significant in the far zone of the cylinder is directly related to $A_{z}$ as

$$
\left[E_{e}^{s}\right]_{\theta}=j \omega A_{z} \cos \theta .
$$

Equation (41) implies that the significant component of the scattered EM wave is the $\theta$ component in a spherical coordinate which origin is located at the center of the cylinder and its polar axis along the axis of the cylinder.

The substitution of (40) and (35) in (41) leads to

$$
\begin{aligned}
& {\left[E_{e}^{s}\right]_{\theta}=\frac{-2 e^{-j \beta_{e} R_{0}}}{\beta_{e} R_{0}}\left[\frac{1-\cos \beta_{e} h}{\cos \beta_{e} h T_{1}-T_{2}}\right]} \\
& \times E_{0}\left[\sin \beta_{e} h \cos \left(\beta_{e} h \sin \theta\right)-(\sin \theta+\cos \theta \cot \theta)\right. \\
& \left.\qquad \cos \beta_{e} h \sin \left(\beta_{e} h \sin \theta\right)\right] .
\end{aligned}
$$

The magnetic field of the scattered EM wave is obtained from a Maxwell equation as

$$
\left[H^{s}\right]_{\phi}=\frac{\sqrt{\xi}}{\zeta_{0}}\left[E_{e}^{s}\right]_{\theta}
$$

The magnetic field is in $\phi$ direction. $\zeta_{0}$ is equal to $120 \pi$ ohms.

The scattered EA wave at a distant point $\left(R_{0}, \theta\right)$ from the center of the cylinder can be determined after $n_{1}$ in (21) is evaluated. With the charge on the cylinder as expressed in (38), $n_{1}$ can be evaluated as

$$
\begin{gathered}
n_{1}=\frac{1}{4 \pi} \frac{\omega_{p}^{2}}{e v_{0}^{2}} \frac{e^{-j \beta_{a} R_{0}}}{R_{0}}\left[\frac{-j \beta_{e}}{\omega}\right] C E_{0} \cos \theta \int_{-h}^{h} \sin \beta_{e} z e^{j \beta_{a} z \sin \theta} d z \\
=\frac{1}{2 \pi} \frac{\omega_{p}^{2}}{e v_{0}^{2}} \frac{e^{-j \beta_{a} R_{0}}}{R_{0}} \frac{\beta_{e}}{\omega} C E_{0} \frac{\cos \theta}{\beta_{e}^{2}-\beta_{a}^{2} \sin ^{2} \theta} \\
\quad \times\left[\beta_{a} \sin \theta \sin \beta_{e} h \cos \left(\beta_{a} h \sin \theta\right)\right. \\
\left.\quad-\beta_{e} \cos \beta_{e} h \sin \left(\beta_{a} h \sin \theta\right)\right]
\end{gathered}
$$

From (20), the radiation term of $\vec{E}_{a}$ which represents the significant component in the far zone of the cylinder can be obtained as

$$
\begin{gathered}
{\left[E_{a}^{s}\right]_{r}=\frac{-j \omega_{p}^{2}}{\left(\omega^{2}-\omega_{p}^{2}\right) \epsilon_{0}}\left(\frac{\beta_{a}}{2 \pi}\right)\left(\frac{\beta_{e}}{\omega}\right) \frac{e^{-j \beta_{a} R_{0}}}{R_{0}} C E_{0} \frac{\cos \theta}{\beta_{e}^{2}-\beta_{a}^{2} \sin ^{2} \theta}} \\
\quad \times\left[\beta_{a} \sin \theta \sin \beta_{e} h \cos \left(\beta_{a} h \sin \theta\right)\right. \\
\left.-\beta_{e} \cos \beta_{e} h \sin \left(\beta_{a} h \sin \theta\right)\right] .
\end{gathered}
$$

The significant component of the electric field of the scattered EA wave is in the radial direction.

The mean induced velocity of electrons of the EA mode can be determined from (14) and (45) as

$$
\begin{aligned}
{\left[V_{a}^{s}\right]_{r}=} & \frac{\omega_{p}^{2}}{\left(\omega^{2}-\omega_{p}^{2}\right)} \frac{\beta_{e}}{e n_{0}} \frac{\beta_{a}}{2 \pi} \frac{e^{-j \beta_{a} R_{0}}}{R_{0}} C E_{0} \frac{\cos \theta}{\beta_{e}^{2}-\beta_{a}^{2} \sin ^{2} \theta} \\
& \times\left[\beta_{a} \sin \theta \sin \beta_{e} h \cos \left(\beta_{a} h \sin \theta\right)\right. \\
& \left.\quad-\beta_{e} \cos \beta_{e} h \sin \left(\beta_{a} h \sin \theta\right)\right] .
\end{aligned}
$$


The significant component of $V_{a}^{s}$ in the far zone of the cylinder is also in the radial direction.

\section{Radar Cross Sections of Cylinder Due to EM and EA Waves}

The power density flow of the scattered EM wave can be represented by a Poynting vector as

$$
\begin{aligned}
S_{e} & =\frac{1}{2}\left[E_{e}^{s}\right]_{\theta}\left[H^{s}\right]_{\phi}^{*} \\
& =\left|\frac{1-\cos \beta_{e} h}{\cos \beta_{e} h T_{1}-T_{2}}\right|^{2} \frac{2 \sqrt{\xi}}{\beta_{e}^{2} R_{0}^{2}} \frac{E_{0}^{2}}{\zeta_{0}}\left[\sin \beta_{e} h \cos \left(\beta_{e} h \sin \theta\right)\right. \\
& \left.-(\sin \theta+\cos \theta \cot \theta) \cos \beta_{e} h \sin \left(\beta_{e} h \sin \theta\right)\right]^{2} .
\end{aligned}
$$

The power density flow of the scattered EA wave has been derived by Field [1956] and can be expressed as

$$
S_{a}=\frac{1}{2} m v_{0}^{2} n_{1}\left[V_{a}^{s}\right]_{r}^{*}
$$

$=\left|\frac{1-\cos \beta_{e} h}{\cos \beta_{e} h T_{1}-T_{2}}\right|^{2} \frac{2 \sqrt{\xi}}{\beta_{a}^{2} R_{0}^{2}}\left(\frac{\omega_{p}^{2}}{\omega^{2}}\right)\left(\frac{c}{v_{0}}\right) \frac{E_{0}^{2}}{\zeta_{0}} \frac{\cos ^{2} \theta}{\left[v_{0}^{2} / c^{2}-\sin ^{2} \theta\right]^{2}}$

$\times\left[\sin \theta \sin \beta_{e} h \cos \left(\beta_{a} h \sin \theta\right)\right.$

$$
\left.-\left(v_{0} / c\right) \cos \beta_{e} h \sin \left(\beta_{a} h \sin \theta\right)\right]^{2} .
$$

The corresponding radar cross sections of the cylinder due to the scattered EM wave is defined as

$$
\sigma_{e}=\lim _{R_{0} \rightarrow \infty} 4 \pi R_{0}^{2}\left[\frac{S_{e}}{E_{0}^{2} \sqrt{\xi / 2 \zeta_{0}}}\right] .
$$

With (47), $\sigma_{e}$ is obtained as

$\sigma_{e}=\left|\frac{1-\cos \beta_{e} h}{\cos \beta_{e} h T_{1}-T_{2}}\right|^{2}\left[\frac{16 \pi}{\beta_{e}^{2}}\right]\left[\sin \beta_{e} h \cos \left(\beta_{e} h \sin \theta\right)\right.$

$\left.-(\sin \theta+\cos \theta \cot \theta) \cos \beta_{e} h \sin \left(\beta_{e} h \sin \theta\right)\right]^{2}$.

The corresponding radar cross section of the cylinder due to the scattered EA wave is defined as

$$
\sigma_{a}=\lim _{R_{0} \rightarrow \infty} 4 \pi R_{0}^{2}\left[\frac{S_{a}}{E_{0}^{2} \sqrt{\xi / 2 \zeta_{0}}}\right] .
$$

With (48), $\sigma_{a}$ is obtained as

$$
\begin{gathered}
\sigma_{a}=\left|\frac{1-\cos \beta_{e} h}{\cos \beta_{e} h T_{1}-T_{2}}\right|^{2}\left[\frac{16 \pi}{\beta_{e}^{2}}\right]\left(\frac{\omega_{p}^{2}}{\omega^{2}}\right)\left(\frac{v_{0}}{c}\right) \frac{\cos ^{2} \theta}{\left[v_{0}^{2} / c^{2}-\sin ^{2} \theta\right]^{2}} \\
\times\left[\sin \theta \sin \beta_{e} h \cos \left(\beta_{a} h \sin \theta\right)\right. \\
\left.\quad-\left(v_{0} / c\right) \cos \beta_{e} h \sin \left(\beta_{a} h \sin \theta\right)\right]^{2} .
\end{gathered}
$$

Physically, $\sigma_{e}$ and $\sigma_{a}$ give the measures of the radar returns from the cylinder in the form of an
$\mathrm{EM}$ and an EA wave. The $\sigma_{e}$ is a slowly varying function of $\theta$ and has a maximum at $\theta=0$ (broadside direction). The $\sigma_{a}$ is a rapidly varying function of $\theta$ and it has the appearance of a group of regularly spaced spikes with two largest maxima near $\theta=0$. The magnitude of $\sigma_{a}$ is much greater than that of $\sigma_{e}$ in the usual cases.

For a numerical example, the case of a cylinder with $\beta_{e} h=\pi / 6 \quad\left(\right.$ or $\left.h=\frac{\lambda_{e}}{12}\right)$ and in a compressible plasma with $\omega_{p}^{2} / \omega^{2}=0.7$ and $v_{0} / c=10^{-3}$ is considered. The $\sigma_{e}$ as a function of $\theta$ is shown graphically in figure 3. The magnitude of $\sigma_{e}$ is normalized by the factor of $\left|\frac{1-\cos \beta_{e} h}{\cos \beta_{e} h T_{1}-T_{2}}\right|^{2}\left[\frac{16 \pi}{\beta_{e}^{2}}\right]$. The shape of $\sigma_{e}$ in figure 3 is similar to that of the radar return from a cylinder in free space. The $\sigma_{a}$ as a function of $\theta$ is shown graphically in figure 4 . The magnitude of $\sigma_{a}$ is normalized by the same factor as in the case of $\sigma_{\epsilon}$. We observe that the magnitude of $\sigma_{a}$ is many orders higher than that of $\sigma_{e}$. The shape of $\sigma_{a}$ in figure 4 consists of a group of bursts with two largest maxima near the center. The appearance of $\sigma_{a}$ would look like a group of spikes if $\sigma_{a}$ is plotted against the axis of $\theta$ with the same scale as that of figure 3 . The interesting point is that the response of $\sigma_{a}$ is concentrated in the range of a very small angle $\theta$.

The total radar cross section of a cylinder is the sum of $\sigma_{e}$ and $\sigma_{a}$. However, since $\sigma_{a}$ is much greater than $\sigma_{e}$ the radar return of the cylinder may be dominated by the contribution of the EA wave.

Kraus et al. [1958a, b, 1960] observed the radar returns of the satellites with a HF signal of $20 \mathrm{Mc} / \mathrm{s}$. If their observations are compared with figure 4, we will find some striking resemblances. First, the appearance of the radar return is very similar and secondly the magnitude of $\sigma_{a}$ exceeds that of $\sigma_{e}$ which corresponds to the physical cross section of the cylinder by many orders of magnitude. This finding may lead someone to suspect that the unusual observations of the satellites made by Kraus et al. [1958a, b, 1960] is due to the excitation of an EA wave in the ionosphere by the satellites.

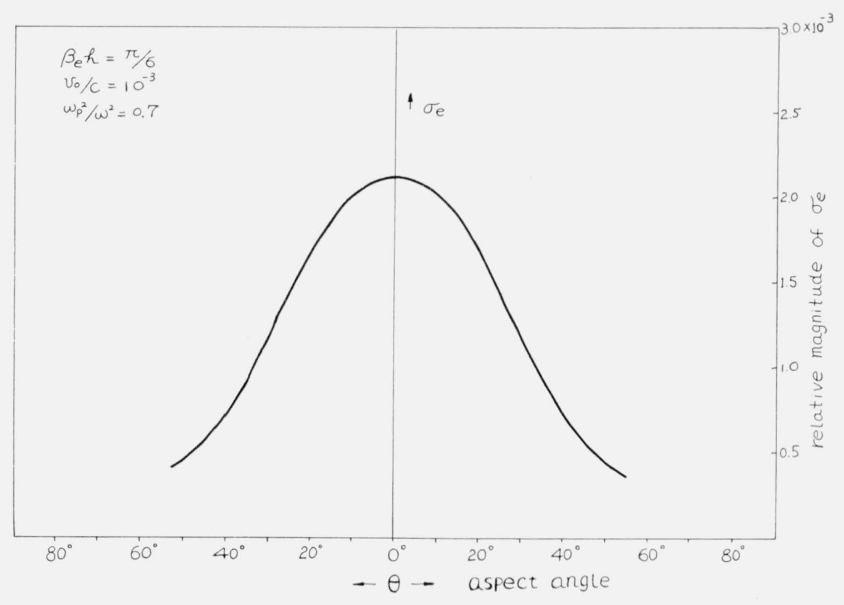

Figure 3. Radar cross section due to $E M$ wave, $\sigma_{\epsilon}$, versus aspect angle $\theta$. 


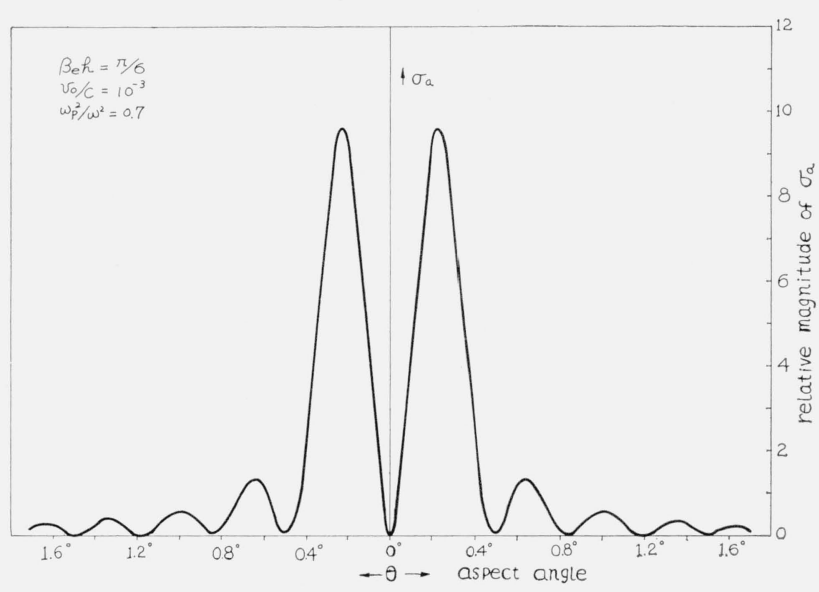

Figure 4. Radar cross section due to $E A$ wave, $\sigma_{\mathrm{a}}$, versus aspect angle $\theta$.

\section{Conversion of EA Wave to EM Wave Across a Density Discontinuity}

In the preceding section we have shown that an EA wave excited by a conducting object in a compressive plasma can greatly enhance the radar cross section of the object. However, for the energy of an EA wave to reach a receiving antenna on the ground the EA wave must be converted to an EM wave to propagate in the air. The mechanism for the conversion of an EA wave to an EM wave has been investigated by Kritz and Mintzer [1960] and Field [1956]. Their results are briefly summarized here. Kritz and Mintzer have shown that the transmission of a longitudinal plasma wave (EA wave) across a plasma density discontinuity such as the lower boundary of the ionosphere can cause transverse EM waves to be formed. When the incident angle of the plasma wave is zero, no EM wave appears but for a small cone of incident angle

$$
\sin \theta \leq \frac{v_{0}}{c} \frac{1}{\sqrt{1-\omega_{p}^{2} / \omega^{2}}}
$$

a portion of a plasma wave will be converted to an EM wave. The conversion coefficient increases as the frequency of incident plasma wave approaches the plasma frequency.

Field also calculated the conversion coefficient, $\gamma$, defined as the ratio of the normal flux in the transmitted EM wave to that in the incident plasma wave at a plasma-vacuum boundary. He obtained an average conversion coefficient as

$$
\gamma_{\mathrm{avg}}=4 \frac{\lambda_{0}}{\lambda_{p}} \frac{3 k T}{m c^{2}}
$$

where $\lambda_{0}$ and $\lambda_{p}$ are the wavelengths of the transmitted EM wave and the incident plasma wave. He also found that the maximum conversion occurs at $\sin \theta=v_{0} / c$. In conclusion, he mentioned that under special condition complete conversion occurs even without a static magnetic field if the temperature of the plasma is very high.

In all, the mechanism for the conversion of an EA wave to an EM wave across the density discontinuity is known and we are quite certain that the EA wave excited by a space vehicle can enhance the radar return and can be detected on the ground.

\section{Conclusion}

It has been shown that a conducting object in the ionosphere can excite an EA wave in addition to an EM wave if it is illuminated by an incident EM wave. The excited EA wave can give a strong enhancement to the radar return of the object. This enhancement is shown to resemble in some ways to the experimental results of the radar return of a satellite. In light of this finding it may be worth while to study the problem more rigorously by choosing a different shape for the object and taking into account the plasma sheath surrounding the object and the effect of the earth's magnetic field, etc.

\section{Appendix}

Determination of $\left[I_{z}(z)\right]_{0}$ from (33).

When we assume that the length of the cylinder is much smaller compared with the wavelength of the incident EM wave, the following condition is implied.

$$
\beta_{e}^{2} h^{2} \sin ^{2} \theta<<1
$$

With (55), (33) can be simplified to

$$
\left(\frac{\partial^{2}}{\partial z^{2}}+\beta_{e}^{2}\right) \int_{-h}^{h}\left[I_{z}\left(z^{\prime}\right)\right]_{0} K_{e}\left(z, z^{\prime}\right) d z^{\prime}=\frac{-j 4 \pi \beta_{e}^{2}}{\omega \mu_{0}} E_{0} \cos \theta
$$

If we write

$$
A_{z}(z)=\frac{\mu_{0}}{4 \pi} \int_{-h}^{h}\left[I_{z}\left(z^{\prime}\right)\right]_{0} K_{e}\left(z, z^{\prime}\right) d z^{\prime}
$$

(56) becomes

$$
\left(\frac{\partial^{2}}{\partial z^{2}}+\beta_{e}^{2}\right) A_{z}(z)=\frac{-j \beta_{e}^{2}}{\omega} E_{0} \cos \theta .
$$

The solution for $A_{z}(z)$ is

$$
A_{z}(z)=\frac{-j}{v_{e}}\left[C_{1} \cos \beta_{e} z+\frac{E_{0}}{\beta_{e}} \cos \theta\right]
$$

where $v_{e}=\frac{1}{\sqrt{\mu_{0} \epsilon_{0} \xi}}$ and $C_{1}$ is an arbitrary constant. 
We can derive an equation from (59) as

$$
\begin{aligned}
A_{z}(z)-A_{z}(h)= & \frac{-j}{v_{e}} \sec \beta_{e} h\left[j v_{e} A_{z}(h)\right. \\
& \left.\quad-\frac{E_{0} \cos \theta}{\beta_{e}}\right]\left(\cos \beta_{e} z-\cos \beta_{e} h\right) .
\end{aligned}
$$

The combination of (57) with (60) gives

$$
\begin{aligned}
& \int_{-h}^{h}\left[I_{z}\left(z^{\prime}\right)\right]_{0} K_{d}\left(z, z^{\prime}\right) d z^{\prime}=\frac{-j 4 \pi}{\zeta} \sec \beta_{e} h \\
& \quad\left(j v_{e} A_{z}(h)-\frac{E_{0} \cos \theta}{\beta_{e}}\right) \times\left(\cos \beta_{e} z-\cos \beta_{e} h\right)
\end{aligned}
$$

where $K_{d}\left(z, z^{\prime}\right)=K_{e}\left(z, z^{\prime}\right)-K_{e}\left(h, z^{\prime}\right)$ and $\zeta=\sqrt{\frac{\mu_{0}}{\epsilon_{0} \xi}}$. If we assume

$$
\left[I_{z}(z)\right]_{0}=C_{2}\left(\cos \beta_{e} z-\cos \beta_{e} h\right)
$$

and substitute $(62)$ in (61), we can determine $C_{2}$ by by letting $z=0$ in (61). Thus $C_{2}$ can be expressed as

$$
C_{2}=\frac{-j 4 \pi}{\zeta_{0} T_{d}}\left[j v_{e} A_{2}(h)-\frac{E_{0} \cos \theta}{\beta_{e}}\right]\left(\sec \beta_{e} h-1\right)
$$

where

$$
T_{c d}=\int_{-h}^{h}\left(\cos \beta_{e} z^{\prime}-\cos \beta_{e} h\right) K_{d}\left(0, z^{\prime}\right) d z^{\prime} .
$$

The $A_{z}(h)$ in (63) is still unknown but it can be evaluated after (62) and (63) are substituted in (57), $A_{z}(h)$ can be obtained as

$$
A_{z}(h)=\frac{j E_{0} \cos \theta}{v_{e} \beta_{e}} \frac{T_{c a}\left(\sec \beta_{e} h-1\right)}{T_{c d}+T_{c a}\left(1-\sec \beta_{e} h\right)}
$$

where

$$
T_{c a}=\int_{-h}^{h}\left(\cos \beta_{e} z^{\prime}-\cos \beta_{e} h\right) K_{e}\left(h, z^{\prime}\right) d z^{\prime} .
$$

The substitution of (63) and (65) in (62) gives the final solution for $\left[I_{z}(z)\right]_{0}$ as

$$
\begin{array}{r}
{\left[I_{z}(z)\right]_{0}=\frac{j 4 \pi}{\zeta \beta_{e}}\left[\frac{1-\cos \beta_{e} h}{T_{c d} \cos \beta_{e} h-T_{c a}\left(1-\cos \beta_{e} h\right)}\right] E_{0}} \\
\quad \times \cos \theta\left(\cos \beta_{e} z-\cos \beta_{e} h\right) .
\end{array}
$$

If the relation of

$$
T_{c d}+T_{c a}=T_{1} \text { and } T_{c a}=T_{2}
$$

are used in (67), the final solution for $\left[I_{z}(z)\right]_{0}$ is obtained as in (34).

\section{References}

Chen, Kun-Mu (1963), Interaction of a radiating source with a plasma, Program Digest, 252-257, Symposium on Antennas and Propagation, Boulder, Colo. 9-11 July 1963, also appearing in full length in Proc. IEE (London) Oct. $1964,1668-1678$.

Cohen, M. H. (1962), Radiation in a plasma, Phys. Rev. 126, $389-404$.

Field, G. B. (1956), Radiation by plasma oscillations, Astrophys. J. 124, No. 3, 555-570.

Hessel, A., and J. Shmoys (1962), Excitation of plasma waves in a homogeneous isotropic plasma by a dipole, Proceedings of the Symposium on Electromagneties and Fluid Dynamics of Gaseous Plasma, 173-184 (Polytechnic Press, Brooklyn, N.Y.).

Kraus, J. E. (1958), Detection of Sputnik I and II by CW reflection, Proc. IRE 46, 611-612.

Kraus, J. R., R. C. Higgy, and J. S. Albus (1958), Observations of the U.S. satellites explores I and III by CW reflection, Proc. IRE 46, 1534

Kraus, J. D., R. C. Higgy, and W. R. Crone (1960), The satellite ionization phenomenon, Proc. IRE 48, 672-678.

Kritz, A. H., and D. Mintzer (1960), Propagation of plasma waves across a density discontinuity, Phys. Rev. 117, No. 2, 382-386.

Wait, J. R. (1964a), Theory of slotted-sphere antenna immersed in a compressive plasma. Part I and II, Radio Sci. J. Res. NBS/USNC-URSI 68D, No. 10, 1127-1136.

Wait, J. R. (1964b), On radiation of electromagnetic and electroacoustic waves in plasma (private communication).

(Paper 69D2-459) 